\title{
Counting Statistics of Photons Produced by Electronic Shot Noise
}

\author{
C. W. J. Beenakker and H. Schomerus \\ Instituut-Lorentz, Universiteit Leiden, P.O. Box 9506, 2300 RA Leiden, The Netherlands
}

(Received 28 August 2000)

\begin{abstract}
A theory is presented for the photodetection statistics of radiation produced by current fluctuations in a phase-coherent conductor. Deviations are found from the Poisson statistics that would result from a classical current. For detection in a narrow frequency interval $\delta \omega$, the photocount distribution has the negative-binomial form of blackbody radiation if $e \delta \omega$ is less than the mean current $\bar{I}$ in the conductor. When electronic localization sets in, $\bar{I}$ drops below $e \delta \omega$ and a different type of super-Poissonian photon statistics results.
\end{abstract}

DOI: $10.1103 /$ PhysRevLett.86.700

Some interesting recent developments in mesoscopic physics have arisen from the interplay with quantum optics [1]. To mention two examples, the Hanbury-Brown-Twiss effect for photons is inspiring the search for its electronic counterpart [2], while single-electron tunneling has been used to create a single-photon turnstile device [3]. An appealing subject for research, in line with these developments, is the study of a mesoscopic conductor through the quantum optical properties of the radiation produced by the current fluctuations. It is a textbook result [4], due to Glauber [5], that a classical current produces photons with Poisson statistics. What is the photon statistics for a fully phase-coherent conductor? That is the fundamental question addressed in this paper.

It is a timely question in view of a recent proposal by Aguado and Kouwenhoven [6] to use photon-assisted tunneling in a device containing two quantum dots in series as a detector for the microwave radiation emitted by a nearby mesoscopic conductor. One such device by itself can give information only on the mean rate of photon production, calculated in Refs. [6-8], but a pair of devices could measure the time-dependent correlations and hence could detect deviations from Poisson statistics due to photon bunching [9].

We will calculate these fluctuations for an idealized model of a photodetector, the same model that leads to the Glauber formula of photodetection theory [10]. In this formula the photocount distribution is expressed as an expectation value of normally ordered photon creation and annihilation operators. (Normal ordering means that all creation operators are brought to the left of the annihilation operators.) We will see that the ordering inherited by the electron current operators involves not only a normal ordering, but in addition an ordering of the incoming current with respect to the outgoing current.

We present a general formula for the variance of the photocount in terms of the transmission and reflection matrices of the conductor. A particularly simple result is obtained in the limit that the frequency interval $\delta \omega$ of the detected radiation is small compared to the mean (particle) current $\bar{I} / e$ through the conductor: The photocount distribution $P(n)$ for a long counting time $\tau$ is then proportional to the negative-binomial coefficient $\left(\begin{array}{c}n+\nu-1 \\ n\end{array}\right)$ (with $\nu=\tau \delta \omega /$
PACS numbers: 73.50.Td, 42.50.Ar, 42.50.Lc, 73.23.-b

$2 \pi \gg 1$ ) [11]. This is the photonic counterpart of the (positive) binomial counting distribution for electrical charge [12-15]. In the localized regime the condition $\delta \omega \ll \bar{I} / e$ breaks down and a different non-Poissonian distribution results.

The starting point of our analysis is an expression for the photocount distribution as a time-ordered expectation value of the electric field operator [10],

$$
\begin{aligned}
P(n) & =\left\langle\mathcal{T}_{ \pm} \frac{1}{n !} W^{n} e^{-W}\right\rangle, \\
W & =\int_{0}^{\infty} d \omega \alpha(\omega) \iint_{0}^{\tau} d t_{-} d t_{+} e^{i \omega\left(t_{+}-t_{-}\right)} E\left(t_{-}\right) E\left(t_{+}\right) .
\end{aligned}
$$

Here $P(n)$ is the probability to detect $n$ photons in a time interval $\tau$ and $E(t)$ is the operator of the detected mode of the electric field in the Heisenberg picture. (We assume for simplicity that a single mode is detected.) The detector has sensitivity $\alpha(\omega)$ at frequency $\omega>0$. The symbol $\mathcal{T}_{ \pm}$ indicates the Keldysh time ordering of the time-dependent operators: times $t_{-}$to the left of times $t_{+}$, earlier $t_{-}$to the left of later $t_{-}$, earlier $t_{+}$to the right of later $t_{+}$.

The Glauber formula is obtained from Eq. (1) by substituting the free-field expression

$$
E_{\text {free }}(t) \propto \int_{0}^{\infty} d \omega\left[a^{\dagger}(\omega) e^{i \omega t}+a(\omega) e^{-i \omega t}\right]
$$

for $E(t)$ and by making the rotating-wave approximation (neglecting $e^{i\left(\omega+\omega^{\prime}\right) t}$, retaining $\left.e^{i\left(\omega-\omega^{\prime}\right) t}\right)$. The time ordering of the electric field then becomes normal ordering of the photon operators $a^{\dagger}, a$. Our goal is instead to go from Eq. (1) to an expression in terms of the electron operators $c^{\dagger}, c$ that constitute the current operator $I(t)$.

The electron and photon degrees of freedom are coupled in the Hamiltonian via a term $-\int d \mathbf{r} \mathbf{j}(\mathbf{r}, t)$. $\mathbf{A}(\mathbf{r}, t)$, where $\mathbf{j}$ is the electron current density operator and $\mathbf{A}$ is the electromagnetic vector potential. This coupling leads to a linear integral relation between $E$ and $I$ :

$$
E(t)=E_{\text {free }}(t)+\int_{-\infty}^{\infty} d t^{\prime} g\left(t-t^{\prime}\right) I\left(t^{\prime}\right) .
$$

The propagator $g(t)$ vanishes for $t<0$ because of causality. (We are neglecting retardation of the electromagnetic radiation.) We assume that electrons and photons are 
uncoupled for $t \rightarrow-\infty$, the photons starting out in the vacuum state. Substitution of Eq. (3) into Eq. (1) leads to a correlator involving the noncommuting operators $E_{\text {free }}$ and $I$. Fleischhauer has shown [16] that the vacuum term $E_{\text {free }}$ may be removed from the correlator if the Keldysh time ordering of $E$ is carried over to $I$ :

$$
\begin{aligned}
W= & \int_{0}^{\infty} d \omega \alpha(\omega) \iint_{0}^{\tau} d t^{\prime} d t^{\prime \prime} e^{i \omega\left(t^{\prime \prime}-t^{\prime}\right)} \iint_{-\infty}^{\infty} d t_{-} d t_{+} \\
& \times g\left(t^{\prime}-t_{-}\right) g\left(t^{\prime \prime}-t_{+}\right) I\left(t_{-}\right) I\left(t_{+}\right) .
\end{aligned}
$$

To leading order in $g$ we may neglect the coupling to the photons in the time dependence of $I(t)$. For free electrons the time dependence is given by [17]

$$
I(t)=\frac{1}{2 \pi} \iint d \varepsilon d \varepsilon^{\prime} e^{i\left(\varepsilon-\varepsilon^{\prime}\right) t} c^{\dagger}(\varepsilon) M\left(\varepsilon, \varepsilon^{\prime}\right) c\left(\varepsilon^{\prime}\right),
$$

$M\left(\varepsilon, \varepsilon^{\prime}\right)=S^{\dagger}(\varepsilon) D S\left(\varepsilon^{\prime}\right)-D$,

$$
D=\left(\begin{array}{cc}
0 & 0 \\
0 & 1
\end{array}\right), \quad S=\left(\begin{array}{cc}
r^{\prime} & t^{\prime} \\
t & r
\end{array}\right)
$$

using units such that $\hbar=1$ and $e=1$. We have introduced the scattering matrix $S(\varepsilon)$ (with $N \times N$ reflection and transmission submatrices $\left.r, r^{\prime}, t, t^{\prime}\right)$ of the $N$ propagating modes at energy $\varepsilon$ (relative to the Fermi energy at $\varepsilon=0$ ). The scattering geometry is illustrated in Fig. 1. The detection matrix $D$ selects the current in one of the two leads, arbitrarily chosen to be the right lead in Eq. (7). The total current $I=I_{\text {out }}-I_{\text {in }}$ is then the difference of the current $I_{\text {out }}$ coming from the left and the current $I_{\text {in }}$ coming from the right. These two currents $I_{\text {out }}$ and $I_{\text {in }}$ are defined as in Eq. (5), with the matrix $M$ replaced by $S^{\dagger} D S$ and $D$, respectively.

The separation of $I$ into outgoing and incoming current operators is convenient because they have simple commutation relations: (i) $I_{\text {out }}(t)$ commutes with $I_{\text {out }}\left(t^{\prime}\right)$; (ii) $I_{\text {in }}(t)$ commutes with $I_{\text {in }}\left(t^{\prime}\right)$; (iii) $I_{\text {out }}(t)$ commutes with $I_{\text {in }}\left(t^{\prime}\right)$ if $t<t^{\prime}$. It follows that Keldysh time ordering of the current operators is the same as an ordering whereby the operators $I_{\text {in }}\left(t_{-}\right)$are moved to the left and $I_{\text {in }}\left(t_{+}\right)$to the right of all other operators - irrespective of the values of the time arguments.

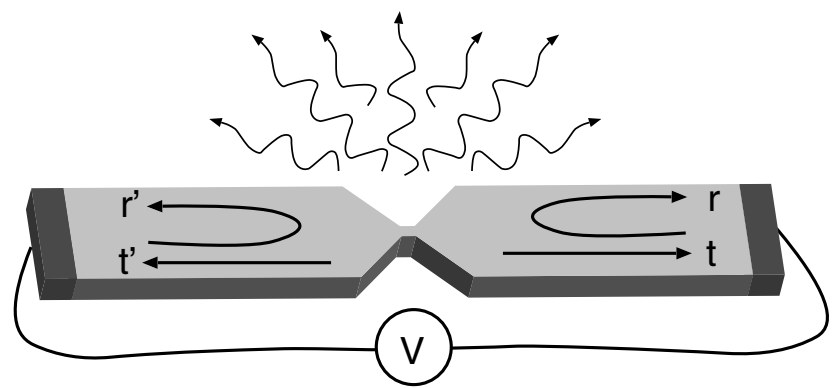

FIG. 1. Illustration of the scattering geometry studied in the text. An electrical current flowing through a constriction emits microwave radiation that is absorbed by a nearby detector.
Now that we have liberated ourselves from the time ordering we are free to take Fourier transforms,

$$
\begin{aligned}
P(n) & =\left\langle\mathcal{O} \frac{1}{n !} W^{n} e^{-W}\right\rangle \\
W & =\int_{0}^{\infty} d \omega \alpha(\omega) U^{\dagger}(\omega) U(\omega), \\
U(\omega) & =\int_{-\infty}^{\infty} \frac{d \omega^{\prime}}{2 \pi} K\left(\omega-\omega^{\prime}\right) g\left(\omega^{\prime}\right)\left[I_{\text {out }}\left(\omega^{\prime}\right)-I_{\text {in }}\left(\omega^{\prime}\right)\right] .
\end{aligned}
$$

The Fourier transforms of $g, I_{\text {out }}, I_{\text {in }}$ are defined as $f(\omega)=$ $\int_{-\infty}^{\infty} d t e^{i \omega t} f(t)$, and we have abbreviated $K(\omega)=$ $\int_{0}^{\tau} d t^{\prime} e^{i \omega t^{\prime}}$. The symbol $\mathcal{O}$ indicates ordering of the current operators from left to right in the order $I_{\text {in }}^{\dagger}, I_{\text {out }}^{\dagger}$, $I_{\text {out }}, I_{\text {in }}$. According to Eq. (5),

$$
\begin{aligned}
I_{\mathrm{out}}(\omega) & =\int d \varepsilon c^{\dagger}(\varepsilon) S^{\dagger}(\varepsilon) D S(\varepsilon+\omega) c(\varepsilon+\omega), \\
I_{\mathrm{in}}(\omega) & =\int d \varepsilon c^{\dagger}(\varepsilon) D c(\varepsilon+\omega) .
\end{aligned}
$$

Equations (8) and (9) form the required relation between the photocount distribution and the electron creation and annihilation operators.

The mean photocount $\bar{n}$ has been studied before [6-8]. To make contact with that work we take the experimentally relevant limit of a long detection time $\tau$. We may then discretize the frequencies as $\omega_{p}=p \times 2 \pi / \tau$. In this discretization the kernel $K$ becomes a Kronecker delta, $K\left(\omega_{p}-\omega_{q}\right)=\tau \delta_{p q} ;$ hence $U\left(\omega_{p}\right)=g\left(\omega_{p}\right) I\left(\omega_{p}\right)$. The factorial moments

$$
\left\langle n^{p}\right\rangle_{\mathrm{f}} \equiv \overline{n(n-1)(n-2) \cdots(n-p+1)}=\left\langle\mathcal{O} W^{p}\right\rangle
$$

of the distribution (8) in the long-time limit take the form [18]

$$
\left\langle n^{p}\right\rangle_{\mathrm{f}}=\left\langle\mathcal{O}\left[\int d \omega \gamma(\omega) I^{\dagger}(\omega) I(\omega)\right]^{p}\right\rangle,
$$

with $\gamma(\omega)=\alpha(\omega)|g(\omega)|^{2}$. For the first moment the ordering operator $\mathcal{O}$ can be omitted and we find

$$
\begin{aligned}
\bar{n} & =\int_{0}^{\infty} d \omega \gamma(\omega)\left\langle I^{\dagger}(\omega) I(\omega)\right\rangle, \\
\left\langle I^{\dagger}(\omega) I(\omega)\right\rangle & =\tau \int_{-\infty}^{\infty} d t e^{i \omega t}\langle I(0) I(t)\rangle,
\end{aligned}
$$

in agreement with Refs. [6-8].

For the double-quantum-dot photodetector of Aguado and Kouwenhoven [6] the response function $\gamma(\omega)$ is sharply peaked at the frequency $\Omega$ of the inelastic tunneling transition, with a width $\delta \omega \ll \Omega$. Its integrated magnitude $\gamma(\Omega) \delta \omega \simeq(Z \Gamma / \Omega)^{2}$ depends on the impedance $Z$ (in units of $h / e^{2}$ ) of the inductive coupling and the ratio of $\Omega$ and the tunnel rate $\Gamma$ between the quantum dots. (Typically, $Z \lesssim 1$ and $\Gamma \ll \Omega$, so that $\gamma \delta \omega \ll 1$.) In that device the inelastic transition can be either upwards or downwards in energy, corresponding, respectively, to the 
absorption or emission of a photon. Here we consider only the case of photodetection by absorption, which is the relevant case for the study of shot noise in the conductor [19].

We now go beyond the first moment to study the entire photocount distribution. We note that $P(n)$ in Eq. (8) would be simply a Poisson distribution,

$$
P_{\text {Poisson }}(n)=\frac{1}{n !} \bar{n}^{n} e^{-\bar{n}},
$$

if the current $I$ would be a classical quantity instead of a quantum mechanical operator. This is in accordance with Glauber's finding [5] that the radiation produced by a classical current is in a coherent state (since a coherent state has Poisson statistics).

To find the deviations from the Poisson distribution due to quantum statistics, let us consider the case of a conductor connecting two electron reservoirs in thermal equilibrium at temperature $T$. The system is brought out of equilibrium by application of a voltage difference $V$ between the left and right reservoirs. Expectation values are given by the Fermi function $f(\varepsilon)=\left(e^{\varepsilon / T}+1\right)^{-1}$,

$$
\left\langle c_{i}^{\dagger}(\varepsilon) c_{j}\left(\varepsilon^{\prime}\right)\right\rangle=\delta_{i j} \delta\left(\varepsilon-\varepsilon^{\prime}\right) f\left(\varepsilon-\mu_{i}\right),
$$

with higher order expectation values obtained by pairwise averaging. The potential $\mu_{i}$ equals $V$ for the left reservoir (mode indices $i=1,2, \ldots, N$ ) and 0 for the right reservoir $(i=N+1, N+2, \ldots, 2 N)$.

For simplicity we restrict ourselves to zero temperature, when $f\left(\varepsilon-\mu_{i}\right)$ becomes the step function $\theta\left(\mu_{i}-\varepsilon\right)$ (equal to 1 for $\varepsilon<\mu_{i}$ and 0 for $\varepsilon>\mu_{i}$ ). The mean and variance of the photocount are then given by

$$
\begin{aligned}
\bar{n}= & \frac{\tau}{2 \pi} \int_{0}^{V} d \omega \mathcal{N}, \\
\operatorname{Var}= & \bar{n}+\frac{\tau}{2 \pi} \int_{0}^{V} d \omega \mathcal{N}^{2} \\
& +\frac{\tau}{2 \pi} \iint_{0}^{V} d \omega d \omega^{\prime} \gamma(\omega) \gamma\left(\omega^{\prime}\right) \\
& \times \int_{0}^{V} d \varepsilon \operatorname{Tr}\left(A_{1}-A_{2}-A_{3}\right) \\
\mathcal{N}= & \gamma(\omega) \int_{\omega}^{V} d \varepsilon \operatorname{Tr} \tau_{\varepsilon} \rho_{\varepsilon-\omega}, \\
A_{1}= & \tau_{\varepsilon}\left(1-\tau_{\varepsilon-\omega}-\tau_{\varepsilon-\omega^{\prime}}\right) \rho_{\varepsilon-\omega-\omega^{\prime}} \\
& \times\left(1-\tau_{\varepsilon-\omega}-\tau_{\varepsilon-\omega^{\prime}}\right) \theta\left(\varepsilon-\omega-\omega^{\prime}\right) \\
A_{2}= & \tau_{\varepsilon} \rho_{\varepsilon-\omega} \tau_{\varepsilon} \rho_{\varepsilon-\omega^{\prime}} \theta(\varepsilon-\omega) \theta\left(\varepsilon-\omega^{\prime}\right), \\
A_{3}= & \rho_{\varepsilon} \tau_{\varepsilon+\omega} \rho_{\varepsilon} \tau_{\varepsilon+\omega^{\prime}} \theta(V-\varepsilon-\omega) \theta\left(V-\varepsilon-\omega^{\prime}\right) .
\end{aligned}
$$

We have abbreviated $\tau_{\varepsilon}=t(\varepsilon) t^{\dagger}(\varepsilon)=1-\rho_{\varepsilon}$. Note that the frequencies $\omega, \omega^{\prime}$ appearing in Eqs. (18)-(21) lie between 0 and $V$.
The formula (16) for the mean photocount is known [6-8]; the result (17) for the variance is new. The first term $\bar{n}$ on the right-hand side corresponds to Poisson statistics. The other terms describe the excess noise, consisting of one term containing a single integral over frequency and three more terms containing double frequency integrals. For narrow-band detection the single frequency integral dominates. More precisely, if $\gamma(\omega)$ is nonzero in a narrow frequency range $\delta \omega \ll V$, then

$$
\bar{n}=\nu \mathcal{N}, \quad \operatorname{Var} n=\bar{n}(1+\bar{n} / \nu),
$$

with $\nu=\tau \delta \omega / 2 \pi$. The noise power is bigger than Poissonian by a factor of $1+\bar{n} / \nu$. The correction terms from the double frequency integrals are smaller by a factor of $\delta \omega / \bar{I}$, with $\bar{I} \propto V \operatorname{Tr} t t^{\dagger}$ the mean electrical current flowing between the reservoirs.

In this regime of narrow-band detection one can also calculate easily the higher order moments of the photocount. The factorial cumulants $\left\langle\left\langle n^{p}\right\rangle\right\rangle_{\mathrm{f}}$ are given by

$$
\left\langle\left\langle n^{p}\right\rangle\right\rangle_{\mathrm{f}}=\nu(p-1) ! \mathcal{N}^{p} .
$$

The probability distribution $P(n)$ can be reconstructed from the factorial cumulants via the generating function $F(\xi)=\sum_{p=1}^{\infty}\left(\xi^{p} / p !\right)\left\langle\left\langle n^{p}\right\rangle\right\rangle_{\mathrm{f}}$, by means of the formula

$$
P(n)=\frac{1}{n !} \lim _{\xi \rightarrow-1} \frac{d^{n}}{d \xi^{n}} e^{F(\xi)}
$$

The probability distribution corresponding to Eq. (23) is

$$
P(n)=\left(\begin{array}{c}
n+\nu-1 \\
n
\end{array}\right) \frac{\mathcal{N}^{n}}{(1+\mathcal{N})^{n+\nu}},
$$

which is the negative-binomial distribution with $\nu$ degrees of freedom. (For noninteger $\nu$ the binomial coefficient should be interpreted as a ratio of gamma functions.) It approaches the Poisson distribution (14) in the limit $\nu \rightarrow \infty$ at fixed $\bar{n}=\nu \mathcal{N}$. The negative-binomial distribution is known in quantum optics as the distribution of blackbody radiation [4]. The role of $\mathcal{N}$ is then played by the Bose function $\left(e^{\omega / T}-1\right)^{-1}$ at the temperature of the blackbody. In both contexts $\mathcal{N}$ is a small parameter, and hence the corrections to Poisson statistics are small: for a blackbody, $\mathcal{N}$ is small because $\omega \gg T$, while for the electrical conductor $\mathcal{N} \simeq \gamma \bar{I} \ll 1$.

We have seen that the negative-binomial distribution results if contributions of order $\delta \omega / \bar{I}$ can be neglected. If the electrical conductor is metallic, it is sufficient that $\delta \omega \ll$ $V$, since the conductance (in units of $e^{2} / h$ ) is greater than 1 in a metal. In the localized regime, on the contrary, the conductance becomes exponentially small and terms of or$\operatorname{der} \delta \omega / \bar{I}$ start playing a role-even if $\delta \omega \ll V$. To illustrate this, let us assume that the transmission through the conductor is so small that only terms linear in $\tau_{\varepsilon}$ need to be retained. This leaves only the term $A_{1}$ in Eq. (17), so that

$$
\operatorname{Var} n=\bar{n}+\frac{\tau}{2 \pi}(\gamma \delta \omega)^{2} \theta(V-2 \omega) \int_{2 \omega}^{V} d \varepsilon \operatorname{Tr} \tau_{\varepsilon} .
$$


More generally, the factorial cumulants are given by

$$
\left\langle\left\langle n^{p}\right\rangle\right\rangle_{\mathrm{f}}=\frac{\tau}{2 \pi}(\gamma \delta \omega)^{p} \theta(V-p \omega) \int_{p \omega}^{V} d \varepsilon \operatorname{Tr} \tau_{\varepsilon} .
$$

The full distribution $P(n)$ can be reconstructed by means of the inversion formula (24) but does not have a simple closed-form expression. We note that the deviations from Poisson statistics are again small because $\gamma \delta \omega \ll 1$.

Much larger deviations can be obtained if coherent radiation from a reference source at frequency $\Omega$ is superimposed prior to detection. Such homodyne detection not only amplifies the deviations from Poisson statistics, it also provides a way to measure the counting distribution of electrical charge. To see this, we note that homodyning amounts to the replacement of the current operator $I(\omega)$ by $I(\omega)+I_{0} \delta(\omega-\Omega)$, where $I_{0}$ is some known classical current (a $c$ number, not an operator). For $I_{0} \gg \bar{I}$ we find from Eq. (11) for the factorial cumulants of the photocount distribution the expression

$$
\left\langle\left\langle n^{p}\right\rangle\right\rangle_{\mathrm{f}}=\delta_{p 1}\left(\tau \gamma I_{0}^{2} / 2 \pi\right)+\left(2 \gamma I_{0}\right)^{p}\left\langle\left\langle Q^{p}\right\rangle\right\rangle,
$$

where $\left\langle\left\langle Q^{p}\right\rangle\right\rangle$ is defined through the generating function

$$
\sum_{p=1}^{\infty} \frac{\xi^{p}}{p !}\left\langle\left\langle Q^{p}\right\rangle\right\rangle=\ln \left\langle e^{-I_{\text {in }}^{\dagger} \xi / 2} e^{I_{\text {out }}^{\dagger} \xi / 2} e^{I_{\text {out }} \xi / 2} e^{-I_{\text {in }} \xi / 2}\right\rangle .
$$

Comparison with Refs. [12,13] shows that $\left\langle\left\langle Q^{p}\right\rangle\right\rangle$ has the interpretation of the cumulant of the charge $Q$ transmitted through the conductor (in units of $e$ ). Levitov and Lesovik [12] proposed to measure the charge counting distribution from the precession of a spin $\frac{1}{2}$ coupled to the current. The photodetection scheme proposed here provides an alternative, and possibly more practical, way to count the charge without breaking the circuit.

In summary, we have presented a solution to the classic problem of the statistics of radiation produced by a fluctuating current. We go beyond the textbook result by considering a fully phase-coherent conductor and find small deviations from the Poisson statistics associated with a classical current source. The deviations might be measured using an array of double-quantum-dot photodetectors [6]. The deviations can be amplified by homodyning, in which case they are directly related to the statistics of the electrical charge transmitted through the conductor [12]. We have given specific results for a conductor between normal reservoirs in thermal equilibrium, but our general formulas can be applied to more special current sources as well. The applications to entangled [20] or superconducting [21,22] electrons seem particularly interesting.

We have benefited from discussions with L.S. Levitov, E. G. Mishchenko, B. A. Muzykantskii, and Yu. V. Nazarov. This work was supported by the Dutch Science Foundation NWO/FOM.
[1] Y. Yamamoto and A. Imamoglu, Mesoscopic Quantum Optics (Wiley, New York, 1999).

[2] For a discussion of the experiments by M. Henny et al. [Science 284, 296 (1999)] and W. D. Oliver et al. [Science 284, 299 (1999)], see M. Büttiker, Science 284, 275 (1999).

[3] J. Kim, O. Benson, H. Kan, and Y. Yamamoto, Nature (London) 397, 500 (1999).

[4] L. Mandel and E. Wolf, Optical Coherence and Quantum Optics (Cambridge University, Cambridge, England, 1995).

[5] R. J. Glauber, Phys. Rev. 131, 2766 (1963).

[6] R. Aguado and L. P. Kouwenhoven, Phys. Rev. Lett. 84, 1986 (2000).

[7] G. B. Lesovik and R. Loosen, JETP Lett. 65, 295 (1997).

[8] U. Gavish, Y. Levinson, and Y. Imry, Phys. Rev. B 62, R10637 (2000).

[9] The double-quantum-dot photodetector has a dead time of $1 / \delta \omega$, set by the width $\hbar \delta \omega$ of the inelastic transition. Photon bunching occurs on the time scale of the inverse bandwidth of the detected radiation, which is also $1 / \delta \omega$. This is why one would need at least two such devices to measure deviations from Poisson statistics due to photon bunching. The theory presented here, appropriate for an array of devices, does not include dead-time effects.

[10] R. J. Glauber, Phys. Rev. Lett. 10, 84 (1963); P. L. Kelley and W. H. Kleiner, Phys. Rev. 136, A316 (1964).

[11] This binomial coefficient is called "negative" because it appears as the expansion coefficient of $1-x$ to a negative power $-\nu$. It counts the number of partitions of $n$ bosons among $\nu$ states.

[12] L. S. Levitov and G. B. Lesovik, JETP Lett. 58, 230 (1993); L. S. Levitov, H. Lee, and G. B. Lesovik, J. Math. Phys. (N.Y.) 37, 4845 (1996).

[13] B. A. Muzykantskii and D. E. Khmelnitskii, Phys. Rev. B 50, 3982 (1994).

[14] Yu. V. Nazarov, Ann. Phys. (Leipzig) 8, 507 (1999).

[15] A. Andreev and A. Kamenev, Phys. Rev. Lett. 85, 1294 (2000).

[16] M. Fleischhauer, J. Phys. A 31, 453 (1998).

[17] M. Büttiker, Phys. Rev. Lett. 65, 2901 (1990).

[18] We have reverted to continuous frequency for ease of notation, but the discretization should be borne in mind when encountering a delta function at zero frequency argument: $\delta\left(\omega-\omega^{\prime}\right) \rightarrow \tau / 2 \pi$ when $\omega \rightarrow \omega^{\prime}$. This ensures that the factorial cumulants $\left\langle\left\langle n^{p}\right\rangle\right\rangle_{\mathrm{f}}$ increase linearly with the detection time $\tau$ in the long-time limit.

[19] The case of photodetection by emission was studied within the framework of Glauber's photodetection theory by L. Mandel, Phys. Rev. 152, 438 (1966). The photocount at low temperatures is then dominated by vacuum fluctuations, so that this mode of operation is less well suited for the study of nonequilibrium fluctuations such as shot noise.

[20] G. Burkard, D. Loss, and E. V. Sukhorukov, Phys. Rev. B 61, R16303 (2000).

[21] J. Torrès and T. Martin, Eur. Phys. J. B 12, 319 (1999).

[22] X. Jehl, M. Sanquer, R. Calemczuk, and D. Mailly, Nature (London) 405, 50 (2000). 